\title{
SEGMENTATION OF BRIGHT REGION OF THE OPTIC DISC FOR EYE DISEASE PREDICTION
}

\author{
Rahul Jadhav and Manish Narnaware \\ Department of Computer Science and Engineering, Walchand College of Engineering, India
}

\begin{abstract}
Eye is a vital organ of vision of the human body. Eyes are used almost in every activity whether in reading, affect developmental learning, working and in other untold ways. But, the eye diseases like Cataracts, Macular degeneration, Retinopathy, Glaucoma etc. gradually influence on the eye and leads to blindness. For the early detection of symptoms of eye diseases the ophthalmologist uses the manual observation method. But, that is time consuming and error prone. In this paper, to save the time and reduce the probability of the error, eye disease prediction approach for Glaucoma is developed. For this eye disease prediction approach firstly, the continuous and non-continuous Blood Vessels are segmented using the Coye Filter Approach. Secondly, the bright region of the Optic Disc is segmented using the MRF and Compensation Factor Method. Finally, the channels intensities of the bright region of Optic Disc is compared with the range of channels intensity of the set of bright region of the healthy Retinal images for prediction of the Glaucoma affection. The range for each channel consist of the intensity value starts from minimum to maximum intensities from the set of healthy Retinal images. For this, the Retinal Fundus image is captured by digital Fundus camera with the field of view between 35o to 50o. The Coye Filter Approach, MRF and Compensation Factor Method is applied for the Diaretdb1 and DRIVE which successfully segment the Blood Vessels as well as Optic Disc and also the eye disease prediction approach is applied for the 10 Glaucoma images which correctly predict for the Glaucoma affection.
\end{abstract}

Keywords:

Retinal Fundus Image, Glaucoma, Optic Disc, Blood Vessels, Retinopathy

\section{INTRODUCTION}

Image segmentation divides image into the multiple portions. The segmentation change's the original representation of the image into more meaningful or purposeful form for easily analyzing the various separated components of the image. Basically, the image segmentation is used for find the objects also detect the shape of that object and boundaries bounded by that objects. This can be achieved by simply labeling each pixels from the image in the manner that, the pixels from the same label value shared certain computed features like intensity, color, texture etc. normally, the Output of the any segmentation algorithm is collection of the separated components or the collection of wrenching out region of interest that is also called the contours. The pixels which are belonging to the particular component have shared the same intensity or color value or texture amongst all there pixels but they are significantly different from the other regions. This paper is totally related to the segmentation of the optic disc, but before moving to further it is necessary to know about the Retinal structure. Following few lines describe the Retinal structure and there important elements.

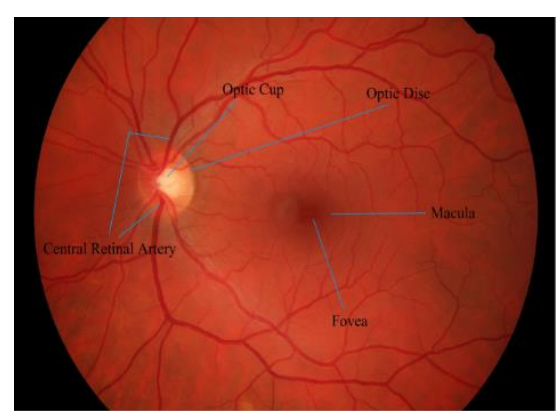

Fig.1. Introduction of Retinal Structure

- Retina: The Retina is nothing but the Fundus region of the eye (part present at the opposite of the pupil). Retina is consist of rods and cons which is very tactful to the light. The retina basically collect the image projected by lenses and convert this image into signal. After converting into the signal this signal is forwarded to the brain using the optic nerve.

- Optic Disc: The Optic Disc is the region of the optic nerve head. This region is circular area with bright pixels on the Retinal image. Basically, the optic disc does not contains any light receptors such as like rods and cons that's why it is also called as Blind Spot. The diameter of the optic disc is about $2 \mathrm{~mm}$. the horizontal and vertical is approximately $1.76 \mathrm{~mm}$ and $1.92 \mathrm{~mm}$ respectively.

- Optic Cup: The Optic Cup is central white part of the Optic Disc. Compare to the Optic Disc the diameter or the size of the Optic Cup is very much less than the Optic Disc. This is main entry point area or exit point for the Optic nerve head and also for the major blood vessels.

- Macula and Fovea: The Macula is located on the center of the retina and the Fovea is the central area of the Macula. This Fovea part is actually the depression on to the retina and it is only consist of the cones (not rods) and also not contains any blood vessels. On the retina the Fovea is located as reddish spot which is approximately $5 \mathrm{~mm}$ from the Optic Disc.

- Central Retinal Artery: The Central Retinal Artery is providing the branches to the internal part of the retina for providing the blood supply to the various major part of the retina. The middle area of the retina where the light rays are focused after passing through pupils and lenses are known as Macula. The central oval shaped area of this Macula is Fovea and this is only the region where Central Retinal Artery is not responsible for supplying the blood vessels. The dimension of the Central Retinal Artery is approximately 160 micrometer. 
- Blood Vessels: there is 2 types of the blood vessels like arteries and veins. The arteries carry the fresh blood from the lungs and hearts to the eye and veins are responsible to away the use blood from the retina to the heart and lungs for refreshing the use blood by the oxygen and other nutrients.

The eye disease like diabetic Retinopathy, Macular degeneration, Cataracts, Glaucoma etc. were ultimately leads towards the blindness. To avoid this blindness there is need to detect the symptoms of this disease as early as possible. For this, ophthalmologist uses the manual observation method which is nothing but observing the Retinal Fundus area by using special dedicated microscope. But this method is more time consuming and also error prone. To avoid the errors and save the time the image segmentation concept is applied on the Retinal Fundus color image. The Retinal Fundus image is captured by various Digital Fundus Camera from $35^{\circ}$ to $50^{\circ}$ FOV and contains the Optic Disc, Optic Cup, Blood Vessels, Macula and Fovea and the outer region of the Retinal Fundus.

In this paper, the Blood Vessels are segmented using the Coye Filter Approach and the Optic Disc from the Retinal image is segmented using the Markov Random Field and also using the Compensation Factor Method. This approaches also preserve the various properties or features of the Blood Vessels and Optic Disc respectively. The image processing toolbox is used for calculating the complete features of the Optic Disc and also the Optic Cup. This extracted features of the Optic Disc and Optic cup is used for detecting the symptoms of the Glaucoma Diseases.

The rest of the paper is organized as follows. Section 2 gives the details of the various segmentation techniques used for the blood vessels and optic disc segmentation. The various approaches such as morphological operations approach, model based approach and pattern based approach etc. are explained in this chapter for blood vessels and optic disc segmentation. Section 3 gives the details of various methodology used for the implementation also gives the importance of this approaches with step by step explanation. This chapters contains the Coye Filter approach for the blood vessels segmentation, MRF and Compensation Factor method is used for the optic disc segmentation and Eye Disease Prediction Approach for detecting the symptoms of Glaucoma Disease. Section 4 contains step by step results of the approaches along with discussion and also analysis. The steps from each approach is step by step explained with input image for the current step and output from the same step. Also, the analysis of the channels intensities for the ROI image is included in the analysis section that is crucial for determining the difference of the channels intensities between the healthy image and Glaucoma affected image. Section 5 include the conclusion of overall paper. This conclusion also shows the importance of some operations which are applied during the implementation of the approaches. Also, include conclusion which are derived from the analysis of the channels of the ROI images.

\section{LITERATURE SURVEY}

The Blood Vessels and Optic Disc Segmentation can be done through various approaches. Amongst these various approaches some popular approaches for the both Blood Vessels and Optic Disc Segmentation such as Model based, Pattern based and Property based morphological approaches etc. are explained below. The section 1 gives the details of the various popular approaches used for the Blood Vessels Segmentation and section 2 lists the various popular techniques used for the Optic Disc Segmentation.

\subsection{BLOOD VESSELS SEGMENTATION APPROACHES}

For the segmentation of the blood vessels there are various approaches is carried out. Amongst these approaches some popular approaches is listed one by one below with detail explanation. For the blood vessel segmentation the mostly used techniques is,

\subsubsection{Tracking Based Approach:}

This Technique covers automated as well as semi-automated tracing. For the semi-automated tracing user have to specify the initial vessel seed point for the blood vessels extraction or for the extraction of the vascular tree. The accuracy of this method is very high that's why, this method is majorly used for segmentation of the arteries which supplies the blood for the heart. Inverse to this method, the automated tracing method automatically select the initial vessel seed point for the blood vessels segmentation. For the selection of the initial vessels seed point the Gaussian function is used to identify the profile of the vessels model and for ultimately detection of the vessel point for segmentation.

\subsubsection{Tracking Based Approach:}

Basically, the Model Based Technique has four approaches, this approaches is listed one by one as following.

- Parametric Models (PM): This approach used the various parameters of the objects. The shape of this blood vessel is like the tubular objects. Basically, this tubular objects are define as overlapping structure of the ellipsoids. For segmenting the blood vessels some of the application apply circular vessel segmentation approach. The method used the parameters of the objects and this parameter is approximated from the input image. The limitation of this approach it can only able to segment the healthy blood vessels which have popular tubular shape. But the vessels which are damaged and having irregular shape are fail to segment by this Parametric Model.

- Template Matching (TM): This method is basically tries to find out the structure model or template from the image and using this template as a preliminary model. This determined template are then used as context that's why it is called as contextual method with top-down approach. For the blood vessel extraction application, the Retinal vascular tree template is defined as a number of nodes connected in portions. This template need to be deformed to fit in the given scene structure. For the deformation the approach specified by the Hidden-Markov-Random-Field is generally used as well as dynamic programing is used for recognizing the vascular tree templates.

- Generalized Cylinders Model: This approach is basically used for recognizing the cylindrical components from the image. As somehow the blood vessels also have the shape of the tubular objects or long cylindrical shape. This method uses the same approach which is used for the Parametric Model. 
- Deformable model: This method is find out the shape of the object by using parametric curve that twist underneath impact of the internal and external pressure [5].

\subsubsection{Neural Network Based Approach:}

This approach is basically used for classification and uses the network of the interconnected fundamental processors (nodes). This processors (nodes) takes the number of input, operate the fundamental operation on that and produce the single output. Therefor each node has assigned a weight and the output function is the weighted total of the inputs. The processors (nodes) weight is read by using the training and used for the identification. This method basically, use the nonlinear categorization boundaries for the training of the network that's why it is more popular in the medical applications for segmentation. There is necessity to select the best training set which acquire all possible properties of the objects so that from this training set the neural network read categorization boundaries into its feature space. The drawback of this method is each time when the new properties are consider, the neural network need to re train for that, and second is it is difficult to compute the network performance [6].

\subsubsection{Miscellaneous Tube Like Object detection Approach:}

This approach is used for extracting the tubular like structure from the image. This miscellaneous class of the approach is only used for the blood vessels segmentation if and only if the blood vessels are in more likely to the tubular structure [7].

\subsection{OPTIC DISC SEGMENTATION APPROACHES}

The basic reason for segmenting the fundamental elements of the Retinal Fundus image like optic disc is to develop superior screening method for detecting the healthiness of the eye. This detection and finding the location of the optic disc is the initial step towards finding the abnormalities as well as lesions in the Retinal image, because the presence of this symptoms may lead to the blindness. So for the early detection of this eye diseases symptoms from the core Retinal structure elements (such as optic disc) segmentation is carried out. For the segmentation of the optic disc there are various approaches are used. Amongst all this approaches some of the mostly used techniques are listed below with detail explanation.

\subsubsection{Model-Based Approaches:}

This method basically uses the template. This templates are matches amongst all the applicants to determine the best applicant amongst all. The Model-Based Approach is initially present by the Osareh et al. [8]. This method generates the gray level templates from taking the mean of the 25 images optic disc regions and the color of this template is normalized using the histogram specification. The center of this optic disc is located by using the morphological filtering and active contour model.

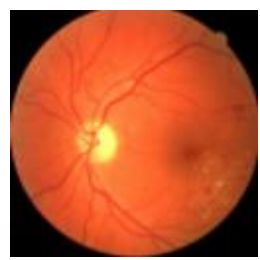

(a) A typical image

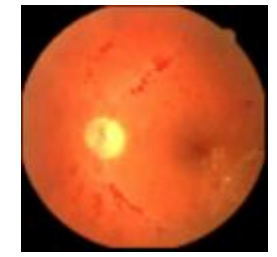

(b) Lab morphology

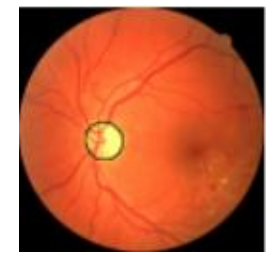

(c) Hand labelled

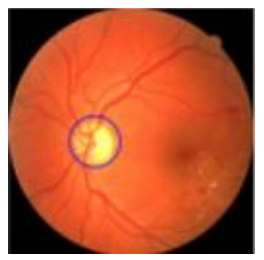

(d) Initial snake

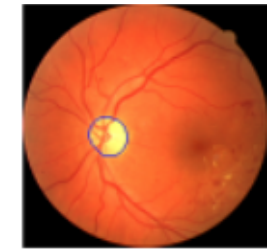

(e) Final snake

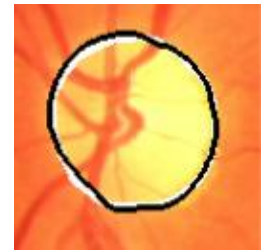

(f) overlay of (c) and (f)
Fig.2. Optic Disc Detection [8]

The $\mathrm{Lu}$ [9] describe the another approach for segmenting the optic disc from the Retinal image. In this technique first, the Retinal background surface was computed from the repeatedly performing the Savitzky-Golay surface smoothing procedure and then optic disc applicant pixels is determined by considering the difference between the Retinal image and the computed Retinal background surface. The accuracy of this approach on the four dataset like DRIVE, DIARETDB0, STARE and DIARETDB1 is respectively $97.50 \%, 98.88 \%, 95.06 \%$, and $99.23 \%$.

\subsubsection{Property-Based Approaches:}

This method is broadly uses the properties which are computed from the image such as the size, color, shape and location of the object. Goldbaue et al. used the three major properties of the optic disc. The first one is bright region of the optic disc, second is convergence of the blood vessels, and third is the arrival and departure of the major blood vessels from the optic disc.

The approach proposed by the Sinthanayothin [10]. Considers the difference between the intensity of the neighboring pixels and the optic disc pixels. To compare the neighboring pixels with the optic disc pixels, this optic disc pixels are grouped together into the window of the optic disc size and this window is compare with all the Retinal pixels. The sensitivity and the specificity of this approach is $99.01 \%$ on the dataset which consist of the 112 TIFF type image.

The Park et al. [11] considers the brightness and the roundness of the optic disc and segment the optic disc from the Retinal image with $90.25 \%$ successful detection.

The Walter and Klein [12] find the centric of the optic disc by considering the optic disc as large bright connected component on to the Retinal image. The approach successfully locate the optic disc from the all images of the local dataset whereas on the STARE dataset the accuracy is $58 \%$.

Zubair et al. [13] proposed the method in which the input image is enhanced using the Contrast Limited Adaptive Histogram Equalization (CLAHE). After enhancing the image the morphological operations like erosion and dilation is performed to remove the non-optic disc region which are not the size of optic disc and to locate the optic disc in the image. From the above the shape of the optic disc is determined and then this optic disc shape preserving image is converted into negative and subtracted from the resized green channel image. The accuracy of this approach on the dataset MESSIDOR is $98.65 \%$. 


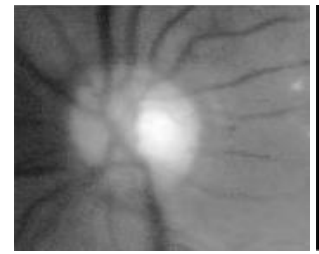

(a)

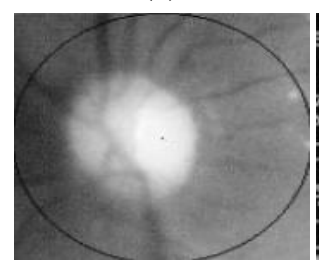

(d)

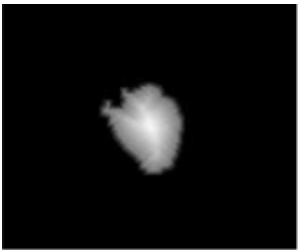

(b)

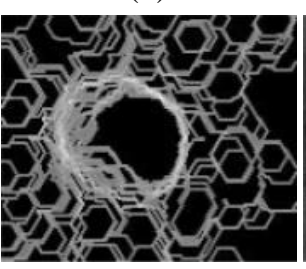

(e)

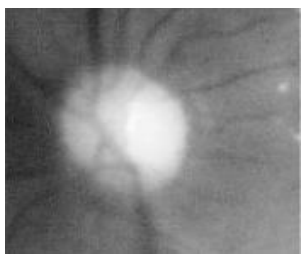

(c)

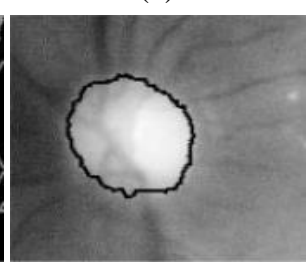

(f)
Fig.3. The Optic Disc Detection (a) Luminance Channel (b) Distance Image of the biggest particle (c) Red Channel (d) Red

Channel imposed marker (e) morphological gradient (f) Segmentation result [10]

\subsubsection{Convergence of Blood Vessels:}

The Ter Haar [14] uses the Hough Transform by two different ways. In first approach the Hough Transform is only exercised on the pixels which are close to the vascular tree extracted image pixels which is extracted by the stall et al. [15] after Hough Transform the morphological dilation process is carried out on to the binary image for increasingly enroll the optic disc applicant. The accuracy of this approach on to the local dataset is about $96.3 \%$ whereas for the STARE dataset it is $71.6 \%$. Another approach where the Hough Transform is exercised for only fuzzy aggregation image consist of brightest $0.35 \%$ generated from the Hoover and Goldbaum. After applying the Hough Transform this contour is again dilated for filling the holes. In this way the optic disc is segmented from the Retinal Fundus image. The success rate of this approach is $97.4 \%$ on the local dataset and $65.4 \%$ on the STARE dataset.

Amongst the different approaches for blood vessel segmentation the contrast enhancement of the gray retinal image using the CLAHE gives the better visibility of the blood vessels which are present on the retina. For the efficient segmentation of the blood vessels, in this paper the Coye Filter approach is used.

Also from the various mentioned approaches for the optic disc segmentation each approach has its own advantages and disadvantages such as tracking based approach has its limitation that it cannot segment the blood vessels which are not in continuous fashion and also for the circular Hough Transform method requires high computation and increase time. The pure property based approach requires to retrain the whole training set for enrolling the new feature of the optic disc. This issues can be slightly overcome by using the Markov Random Field and Compensation Factor Method. The result of this images is also more improve due to use of the Contrast Limited Adaptive Histogram Equalization (CLAHE) as well as it extract the approximate correct bright region of the optic disc. Also this methods are less complex and requires minimum execution time with better accuracy.

\section{SEGMENTATION METHODOLOGY}

In this paper, Blood Vessels Segmentation is carried out by using the Coye Filter approach for segmenting the continuous as well as non-continuous Blood Vessels and MRF and Compensation Factor Method is used for the segmenting the bright region from the Optic Disc. The Eye Disease Prediction Approach is used for detecting the symptoms of the Glaucoma from the input Retinal image. These approaches are illustrated as below.

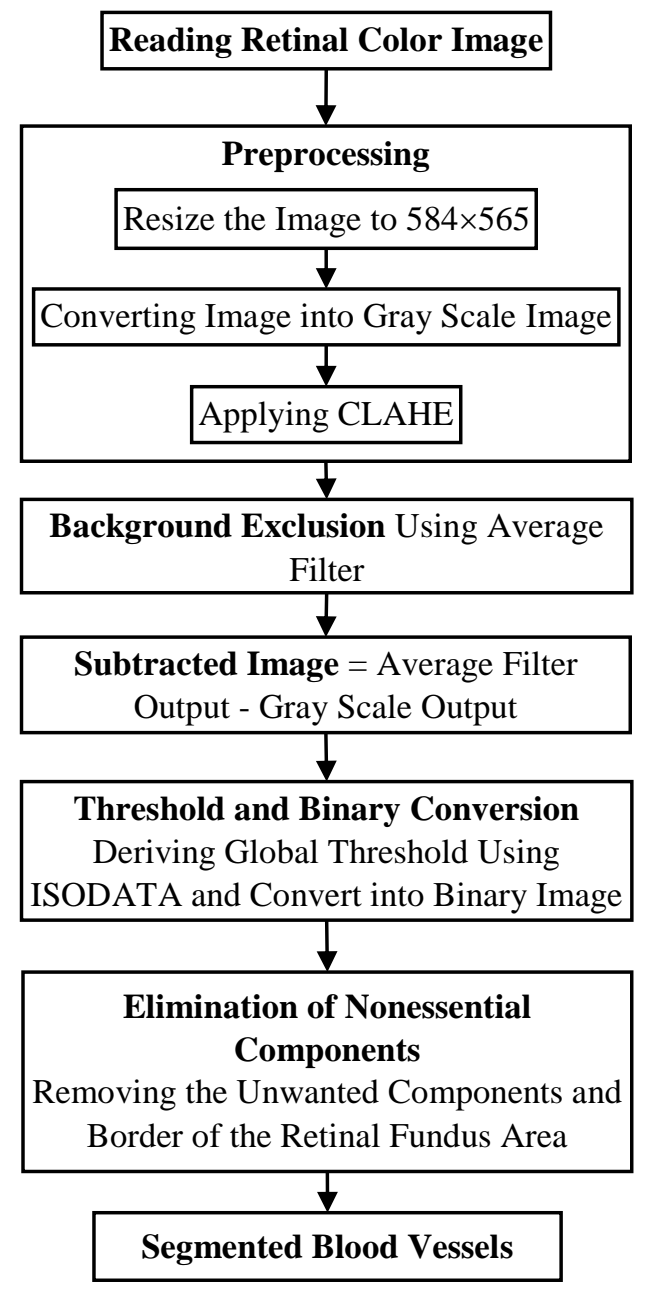

Fig.4. Blood Vessels Segmentation [2]

\subsection{BLOOD VESSELS SEGMENTATIONS METHODOLOGY USING COYE FILTER}

Following are the steps for blood vessels segmentation from the Retinal Fundus image using Coye Filter approach [2] along with the flowchart shown in the figure.

- Reading Retinal Color Image: The input to this approach is 2D color Retinal Fundus image which include the optic disc and blood vessels. This Retinal Fundus image contains the various core Retinal elements such as optic disc, blood vessels, macula, Optic Nerve and fovea.

- Preprocessing: In the preprocessing module, the Retinal Fundus image is resized to the $584 \times 565$ pixels. After resizing, the image is converted into the grayscale image. Further, the converted gray scale image is enhanced using 
Contrast Limited Adaptive Histogram Equalization (CLAHE). This techniques breaks the image into the specified size of the tiles and then perform the contrast enhancement. For this tiles transformation function is computed and then contrast limiting procedure is applied. The raise Contrast of the pel is defined as transformation function gradients. This is corresponds to the neighborhoods cumulative distribution functions (CDF) gradients.

The CLAHE controls the amplification process by hooking the histogram at prior level of intensity before computation of the cumulative distribution function. This also controls the slope of the $\mathrm{CDF}$ and therefore of the transformation function. In this way, the over amplification of the noise is reduced [4].

- Background Exclusion: In this module, the Average filter is applied onto the contrast enhanced image for the background exclusion.

The vector of the contrast enhanced image values is taken as input and compute the average value for each value of the vector. The output vector has same size and shape as previous one. The Average Filter is given in Eq.(1). A moving-average filter slides a window of length along the data, computing averages of the data contained in each window. The following difference equation defines a moving average filter of a vector $x$, window size $=[99]$.

$$
y(n)=\frac{1}{W_{S}}\left(x(n)+x(n-1)+, \ldots,+x\left(n\left(W_{S}-1\right)\right)\right)
$$

where, $W_{S}$ is the window size. As provided by [3].

- Subtracted Image: This image is generated by subtracting the grayscale module output from Average filter output. That is nothing but, Subtraction = Average Filtered Image gray scaled image. The Subtraction image then contain all blood vessels components with some unwanted small components that are may or may not be the part of the blood vessels.

- Threshold and Binary conversion: The ISODATA method is used for computing global threshold using the iterative isodata method. This computed threshold (LEVEL) is used for converting the intensity image into binary image. The LEVEL is normalized intensity value that lies in the range of 0 and 1 . In this technique the histogram is divided into two parts with starting threshold value as like $0=2 B$ 1, maximum dynamic range half. From this, the sample mean $\left(m_{f}, 0\right)$ for the foreground pixels gray value and sample mean $\left(m_{b}, 0\right)$ for the background pixels gray value is computed. A new threshold 1 is now computed as average of this two sample means. The process iterated until the value of the threshold does not change any more. After getting the global threshold (LEVEL) adjusting value is subtracted with this threshold for getting the effective binary conversion of the blood vessels pixels as high intensity value 1 and background pixels as value 0 . The adjusting value is derived by repeatedly examining the output of the binary conversion with sample adjusting value. The most effective binary converted image is consider with its adjusting value.

- Elimination of nonessential components: After successful conversion from gray scale to binary of the Retinal Fundus image. The binary image now contains the blood vessels components as well as unwanted components. To remove this unwanted components and keep the main arcade, the morphological binary image area open operation is applied with specified threshold. The components which consist of the number of pixels greater than threshold is keep as it is, but the components which consist of the number of pixels less than threshold is converted into background pixels by changing that pixels intensity from 1 to 0 . The border of the Retinal Fundus area is also removed by using the bw clear border method.

- Segmented Blood Vessels: The output image of the previous step 6 contains the purely segmented blood vessels. This blood vessels are defined by the active pixels (the pixels having intensity 1) and the background is defined by passive pixels (pixels having intensity 0 ) from the binary image.

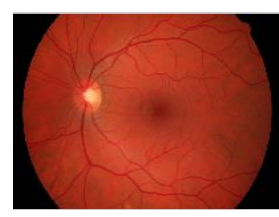

(a)

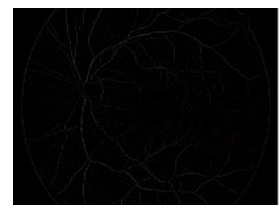

(d)

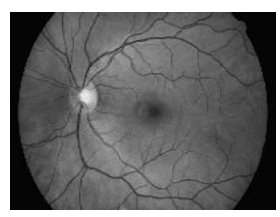

(b)

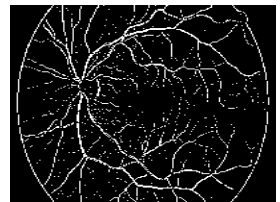

(e)

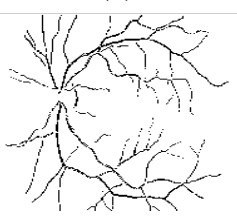

(g)

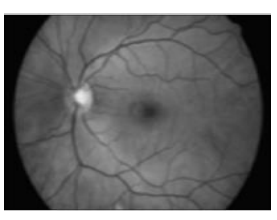

(c)

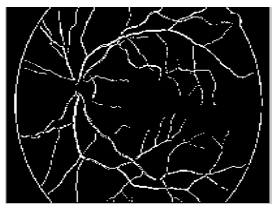

(f)
Fig.5.(a) Input Image, (b) CLAHE Output, (c) Average Filter Output, (d) Subtracted Image, (e) Binary conversion, (f) small elements free Output, (g) Segmented Blood Vessels

\subsection{OPTIC DISC SEGMENTATION}

According to literature survey, there are various methods available for the Optic Disc Segmentation process. However considering the properties of the convergence of the blood vessels and the bright pixels of the optic disc [1], following two methods which have been used in this paper are discussed below.

\subsubsection{Markov Random Field Segmentation Technique:}

Following are the steps for optic disc segmentation from the Retinal Fundus image along with the flowchart shown in the figure.

- Reading Retinal Color Image: The input to this approach is 2D color Retinal Fundus image. This Retinal Fundus image contains the various core Retinal elements such as Optic Disc, Blood Vessels, Macula, Optic Nerve and Fovea.

- Preprocessing: This method takes the input as 2-D color Retinal Fundus image of any size. The Preprocessing module consist of the four step, First the image is resized to the $250 \times 250$ matrix using the cubic interpolation with antialiasing parameter. The antialiasing method is used to perform the antialiasing on the shorten image. 


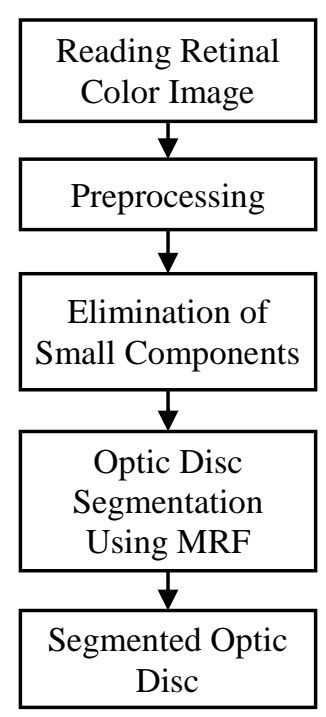

Fig.6. Optic Disc Segmentation using MRF Technique [1]

Secondly, the green channel of the image is extracted. After extracting the green channel at third step, this green channel extracted image is enhanced using the contrast limited adaptive histogram equalization method. The method description is already specified above in the process of blood vessel segmentation for contrast enhancement point. At the fourth step, the contrast enhanced image is filter using the wiener2 filter for reducing the effect of the constant power additive noise. This method based on the statistics calculated by each pixel neighborhood for processing. This statistics contains the mean and standard deviation of the local image. The size of the neighborhood window is $3 \times 3$ square of the pixel. The additive noise is the Gaussian white noise. The details about the wiener2 filter is explained below, wiener2 estimates the local mean and variance around each pixel

$$
\mu=\frac{1}{N M} \sum_{n_{1}, n_{2} \in \eta} a\left(n_{1}, n_{2}\right)
$$

and

$$
\sigma^{2}=\frac{1}{N M} \sum_{n_{1}, n_{2} \in \eta} a^{2}\left(n_{1}, n_{2}\right)-\mu^{2}
$$

where, $\mu$ the $N \times M$ local neighborhood of each pixel is in the image A.

Wiener2 then creates a pixel wise Wiener filter using these estimates,

$$
I\left(n_{1}, n_{2}\right)=\mu+\frac{\sigma^{2}-v^{2}}{\sigma^{2}}(a-\mu)
$$

where, $\sigma$ is the noise variance if the noise variance is not given, wiener2 uses the average of all the local estimated variances [16].

- Elimination of small components: The remove tag module use the output of the preprocessing module. In this, first the preprocessed image is converted into the binary image. This binary image contains number of components that are indicated using the group of the bright pixels having high intensity that is 1 . This components are then labeled. Due to the large size of the optic disc in the binary image, the optic disc in the binary image is indicated by the collection of the

large number of high intensity pixels, so for that there is need to remove the small components from the binary image for reducing the unnecessary processing of that small components. To remove this small components and keeping the only large ellipse shaped object, the threshold is find out by repeatedly performing the morphological open process on the binary image. After getting the threshold, the components which are consist of the number of high intensity pixels greater than threshold is only preserve. The components which are consist of the number of high intensity pixels less than the threshold are converted into the background pixels. Now the image not contains the components less than derived threshold. This image is then converted into the gray scale image. The value for the threshold is 1000 pixels.

- Optic Disc Segmentation using MRF: The module takes the output of the remove tag module as input. After removing the tag, this image is again enhanced using the contrast limited adaptive histogram equalization method and converted into the binary image with new threshold. This new threshold is derived by repeatedly experimenting the process of binary conversion for getting the expected binary image. This binary image then contains some unnecessary small components, this small components are removed by using the bwareaopen() method. Now this binary image contains only large components. From that large components we need to find the oval shaped components. For that, we need to perform the morphological imopen() operation. This morphological open operation is performed using the 9 pixels radius disk shaped structuring element. After getting the oval shape components this components are labeled and centroids of this components are preserved. After getting the centroid of each oval shaped components we used the Hidden Markov Random Field- Expectation Maximization Algorithm. This method is used for 2D image segmentation using HMRF-EM framework.

The HMRF-EM algorithm [18] is used to purify the output of the segmentation which are obtained from various others segmentation algorithm. For getting the accurate segmentation from the complex images some other higher level of features are used instead of using the normal pixel intensities and some leading algorithm is used for producing the starting labels. After labelling the components of the image, the components which Has large oval area amongst the all components is selected and this is nothing but the segmented optic disc from the Retinal image using the Markov random field method. This output is nothing but the binary component which has the intensity either 0 or 1 .

- Segmented Optic Disc: The resulting binary image of previous step contains the convex hull of the optic disc. The region of the optic disc is consist of the active pixels (which has the intensity 1) and other region excluding the optic disc is consist of the passive pixels (which has the intensity 0 ).

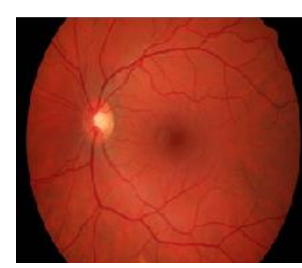

(a)

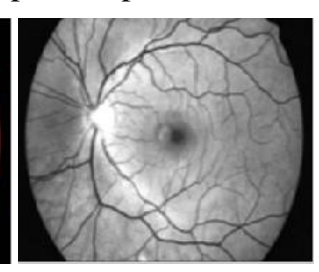

(b)

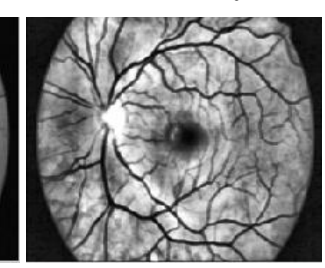

(c) 


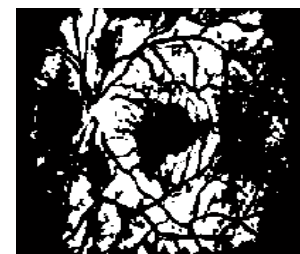

(d)

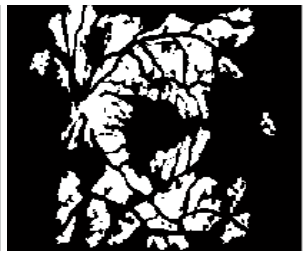

(e)

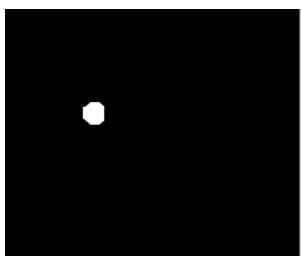

$(\mathrm{g})$

Fig.7.(a) Input Image, (b) Wiener2 Output, (c) CLAHE Output, (d) Binary Image, (e) Small components free Output,

(f) Morphological open process with disc structuring elements Output, (g) Segmented Optic Disc

\subsubsection{Optic Disc Segmentation using Compensation Factor Method:}

Following are the steps for optic disc segmentation from the Retinal Fundus image along with the flowchart shown in the figure.

- Reading Retinal Color Image: The Compensation factor method takes the 2d Retinal Fundus image. This Retinal Fundus image contains the various core Retinal elements such as Optic Disc, Blood Vessels, Macula, Optic nerve and Fovea.

- Channels Extraction: After getting the input image, all channels of this input image is extracted.

- CF Calculation: After extracting all three channels from the input image the mean for these channels is calculated. Amongst from all this calculated mean, the minimum mean value is considered for further process. After getting the minimum mean, the minimum mean is divided by each calculated mean for the respective channels. In this way the compensation factor for all channels (Red, Green and Blue) is calculated respectively.

- Channels intensity reduction: After calculating the compensation factor for each channel, the respective compensation factor is multiplied with the corresponding channel for reducing the effect of the channel. By using this approach we are able to reduce the effect of all the channels from the image.

- Red, Green Channels Enhancement: Due to performing the above steps, we have converted the input image into the neutral form. After converting the image into the neutral form only the red channels and green channels of the image is enhanced to the level $=5$. This levels are in the form of 2's power. The value for the certain level is determined by repeatedly performing the process and examining the output for different values of various level.

- Extraction and Plotting Optic Disc: Every pixel of the already segmented binary image of optic disc is multiplied with the channels intensity reduction output. The resulting image is then converted into the binary image and the perimeter of the optic disc contour is computed. This computed perimeter of the optic disc is plotted onto the enhanced image resulted from the previous step.

- Segmented Optic Disc: This image contains the border around the bright region of the optic disc in the resulting Retinal Fundus image. This is color image of the Retinal Fundus with plotted optic disc perimeter.

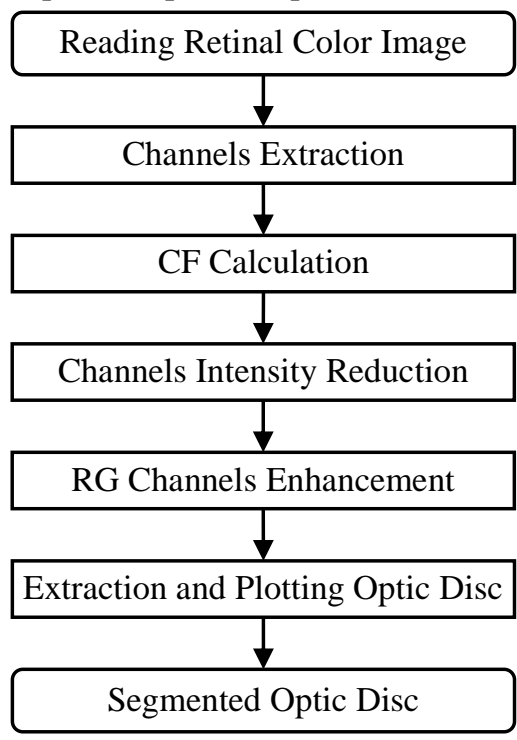

Fig.8. Compensation Factor Method [1]

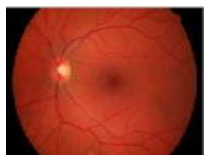

(a)

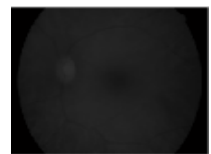

(e)

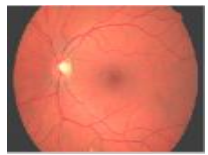

(i)

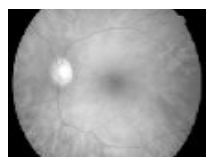

(b)

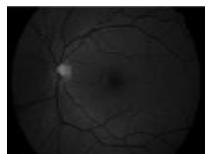

(f)

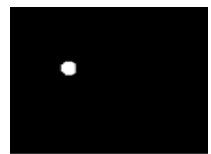

(j)

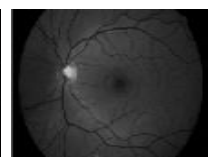

(c)

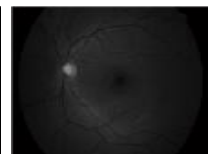

$(\mathrm{g})$

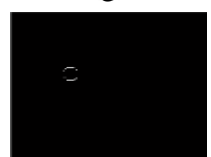

(k)

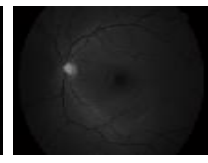

(d)

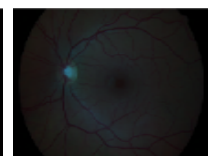

(h)

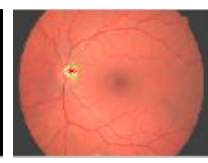

(1)
Fig.9.(a) Input Image, (b) Red Channels, (c) Green Channels, (d) Blue Channels, (e) Reduced Red, (f) Reduced Green, (g) Reduced Blue (h) Reduced Image, (i) Red and Green enhanced Image, (j) MRF output, (k) Perimeter of MRF,

(l) Compensated Output

\subsubsection{Approach for the Eye Disease Prediction:}

Basically this approach is used for locating the symptoms of Glaucoma class of disease. There are various types of eye diseases such as diabetic Retinopathy, Macular degeneration, retinitis, cataracts etc. and each of this eye disease have unique symptoms. In this way, the Glaucoma also has unique symptoms on the retina. In the Glaucoma class of eye disease, due to the internal eye pressure, the optic nerve which connects the eye with brain is damages this ultimately lead to blindness in slow manner. The 
following figure Glaucoma symptoms shows the normal as well as Glaucoma affected Retina.

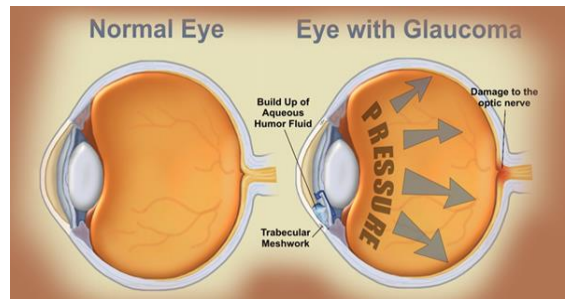

Fig.10. Glaucoma symptoms

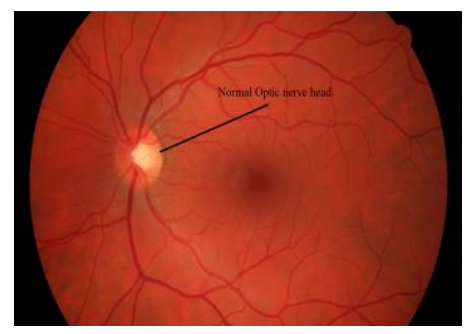

Fig.11. Normal Retinal Image

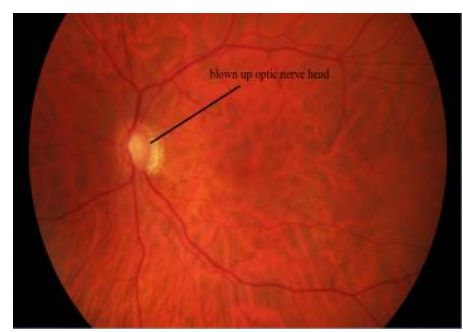

Fig.12. Glaucoma affected Retinal Images

Due to the internal pressure of the retina the optic nerve head is get blown up that is visible in the figure Glaucoma affected Retinal image as compare to the healthy retina which is shown in the figure Normal Retinal Image. For the early detection of the symptoms of the Glaucoma such as like expansion and blow up of the optic nerve head (optic disc). The procedure of the Glaucoma detection only interested in the optic disc region so for that, only region of interest image which contains the optic disc region is provided for this module. This ROI image is computed by the multiplication of the output image of Red, Green Channel Enhancement process with the gray scale image of already segmented optic disc by MRF method. After getting the Region of Interest image the following steps are applied on the input ROI image for the Glaucoma detection.

- Constructing ROI image: This approach is apply on to the only segmented optic disc region. The input for this approach is segmented optic disc region. For to get this input image which contains only the segmented optic disc region in RGB color format the convolution is worked out between the gray scale contour of the segmented bright region of the optic disc and the Red and Green Channels enhanced image as shown in the following figure Region of Interest

- Channels Extraction: All channels of the ROI image is extracted for the further comparison.

- Extraction of sample from bright region of Optic Disc: After getting the region of interest, the $10 \%$ brightest pixels from this region is extracted using the manually specified pixels. After getting the maximum contour of the bright pixels sample, the properties of this contour is computed such as maximum intensity of the red, green, blue channels, area, diameter, major axis length, minor axis length of this contour is computed. The manually specified pixels and the contour for the specified pixels are shown in the following figure.

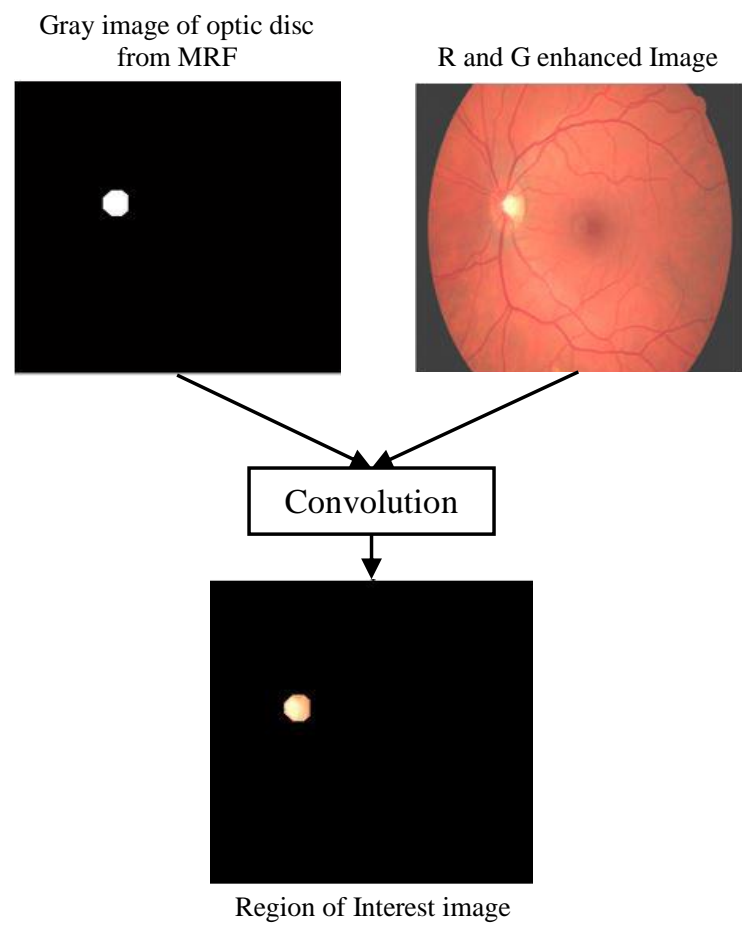

Fig.13. Region of Interest

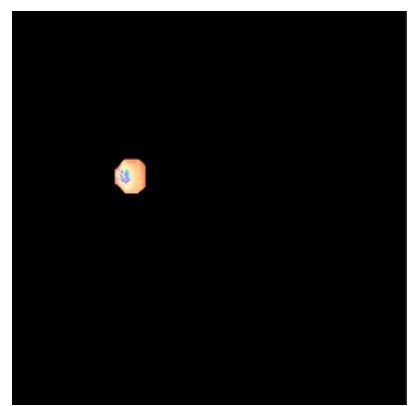

Fig.14. Manually specified of Bright pixels

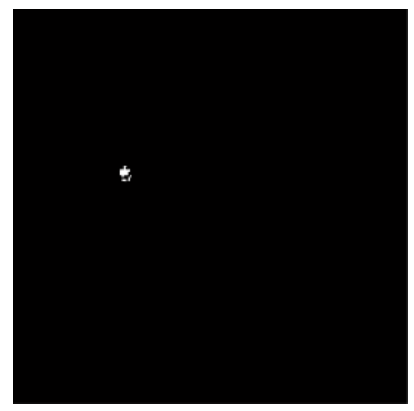

Fig.15. Contour the specified pixels 
- Computing properties for extracted sample: The followings properties are derived for the manually specified pixels and the computed binary contour for that specified pixels which are shown in the $3^{\text {rd }}$ step that is Extraction of sample from bright region of Optic Disc.

Table.1. Extracted Feature of the Contour of the specified pixels

\begin{tabular}{|c|c|}
\hline Properties & Value \\
\hline Maximum Red Channel intensity & 255 \\
\hline Maximum Green Channel intensity & 255 \\
\hline Maximum Blue Channel intensity & 207 \\
\hline Maximum area & 26 \\
\hline Maximum diameter & 5.75 \\
\hline Maximum major axis length & 6.66 \\
\hline Maximum minor axis length & 5.66 \\
\hline
\end{tabular}

After computing the properties, this computed properties is compared with already computed properties from 10 healthy Retinal images for prediction of Glaucoma symptoms. The previously calculated properties for 10 normal healthy Retinal images is as follows.

Table.2. Extracted Feature of the 10 healthy retinal fundus image

\begin{tabular}{|c|c|}
\hline Properties & Value \\
\hline Red intensity from 10 healthy retinal image & 255 \\
\hline Green intensity from 10 healthy retinal image & 255 \\
\hline Minimum Blue intensity from 10 healthy retinal image & 166 \\
\hline Maximum Blue intensity from 10 healthy retinal image & 255 \\
\hline Mean of area from 10 retinal image & 53 \\
\hline Mean of major axis length from 10 retinal image & 10.57 \\
\hline Mean of minor axis length from 10 retinal image & 6.47 \\
\hline Mean of diameter from 10 retinal image & 7.73 \\
\hline
\end{tabular}

Mainly the Glaucoma effect on the region of bright pixels from the optic disc. In this case, the size of the bright region of the optic disc is increases but the intensity of this pixels is decreases with comparison of the healthy Retinal image. Comparison based on calculated properties with precompiled values for Eye Disease Prediction.

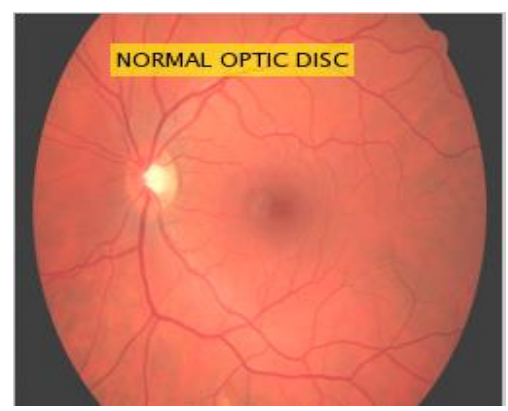

Fig.16. Final result

The input image is categorized as normal healthy image, because, the maximum intensity of red, green, and blue channels of ROI of this image is lies between the precompiled maximum and minimum intensities of the healthy Retinal images. Also the various parameter is also compared but the intensity comparison is crucial for this approach.

- For the Glaucoma affected Retinal Image: The procedure for the Glaucoma affected Retinal image is as following. The same procedure is applied for the Glaucoma affected Optic Disc. First, the Blood Vessels Segmentation is carried out on the input image then, bright region of the Optic Disc is segmented and this ROI image is given input to the Eye Disease Prediction Approach.

- Blood Vessels Segmentation using Coye Filter Approach:

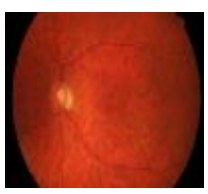

(a)

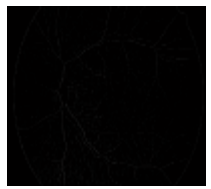

(d)

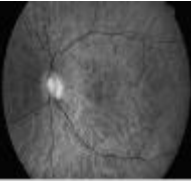

(b)

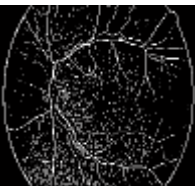

(e)

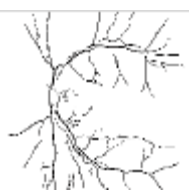

(g)

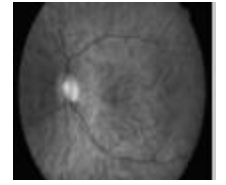

(c)

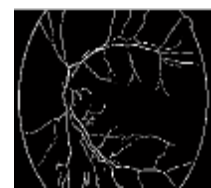

(f)
Fig.17.(a) Input Image, (b) CLAHE Output, (c) Average Filter Output, (d) Subtracted Image, (e) Binary conversion, (f) small elements free Output, (g) Segmented Blood Vessels

\section{- Optic Disc Segmentation using MRF:}

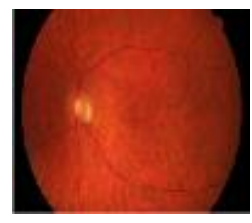

(a)

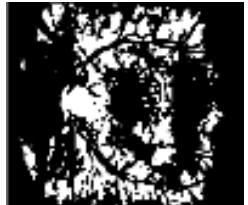

(d)

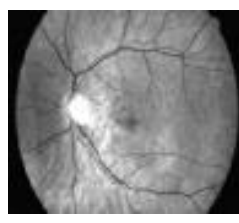

(b)

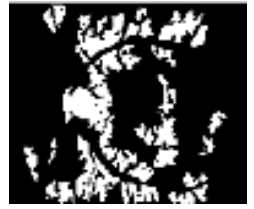

(e)

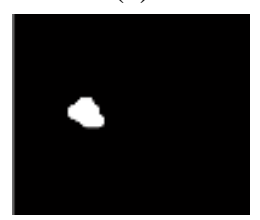

(g)

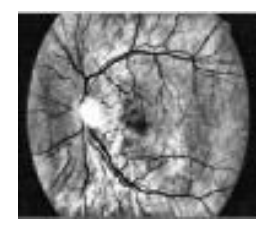

(c)

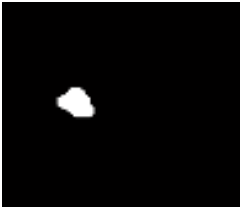

(f)
Fig.18.(a) Input Image, (b) Wiener2 Output, (c) CLAHE Output, (d) Binary Image, (e) small components free Output,

(f) morphological open process with Disc structuring elements Output, (f) Segmented Optic Disc 


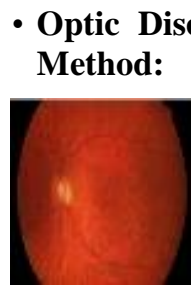

(a)

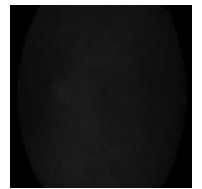

(e)

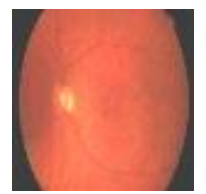

(i)

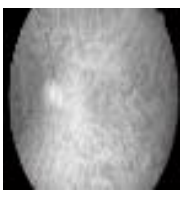

(b)

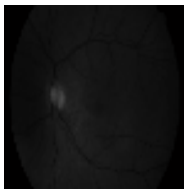

(f)

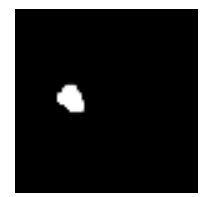

(j)

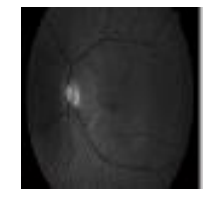

(c)

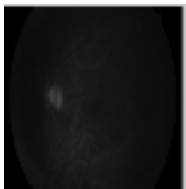

(g)

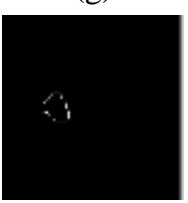

(k)

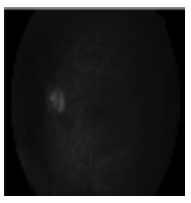

(d)

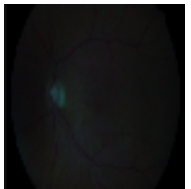

(h)

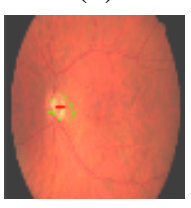

(1)
Fig.19.(a) Input Image, (b) Red Channels, (c) Green Channels,

(d) Blue Channels, (e) reduced Red, (f) reduced Green,

(g) reduced Blue $(\mathrm{h})$ reduced Image, (i) Red and Green enhanced Image, (j) MRF output, (k) perimeter of MRF, (l) Compensated Output

- Applying Eye Dieses Prediction approach for Glaucoma on New image: The following figure's shows the ROI input image, manually specified bright region from that ROI image, and the computed binary contour for that ROI image respectively for the new input image.

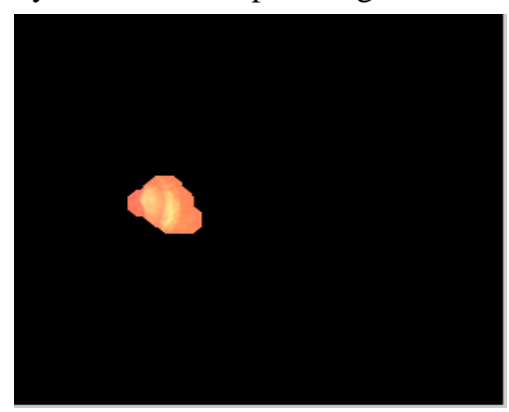

Fig.20. Input image of ROI

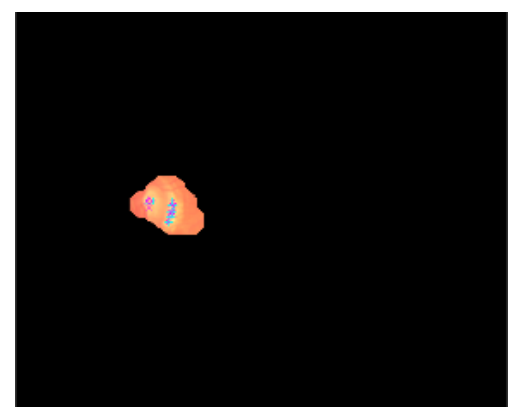

Fig.21. Manually specified bright pixels

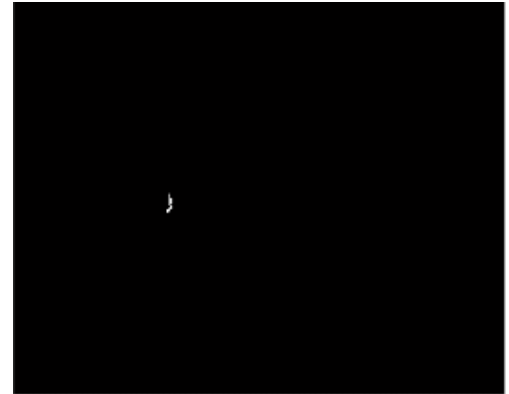

Fig.22. Contour of specified pixels

The properties such as maximum red, green and blue channel intensities are calculated from the manually specified bright pixels. Also the properties such as area, diameter, and maximum major and minor axis length are calculated from the computed binary contour of specified pixels.

Table.3. Extracted Feature of the Contour of the specified pixels

\begin{tabular}{|c|c|}
\hline Properties & Values \\
\hline $\begin{array}{c}\text { Maximum Red Channels intensity from manually } \\
\text { Specified bright pixSels }\end{array}$ & 255 \\
\hline $\begin{array}{c}\text { Maximum Green Channels intensity from manually } \\
\text { Specified bright pixels }\end{array}$ & 231 \\
\hline $\begin{array}{c}\text { Maximum Blue Channels intensity from manually } \\
\text { Specified bright pixels }\end{array}$ & 148 \\
\hline Area of Maximum component & 18 \\
\hline Major Axis Length & 12.84 \\
\hline Minor Axis Length & 4.78 \\
\hline Diameter & \\
\hline
\end{tabular}

The new input image is categorized as glaucoma affected optic disc retinal image, because The maximum intensity of red, green, and blue channels of ROI of this image is not lies between the precompiled maximum and minimum intensities of the healthy retinal images.

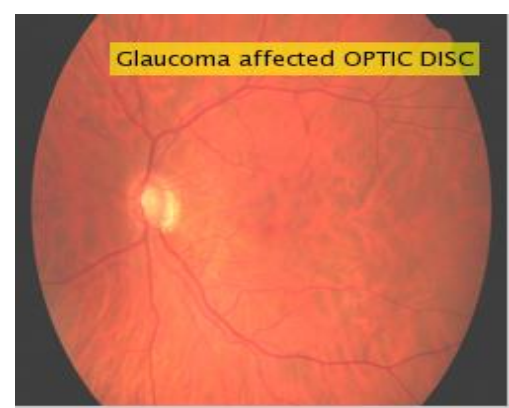

Fig.23. Final result

Also the various parameter is also compared but the intensity comparison is crucial for this approach.

\section{RESULT AND ANALYSIS}

The Blood Vessels Segmentation Coye Filter Approach is applied on the 5 different Retinal images datasets. The result of this 5 datasets are one by one explained below with details of the global threshold calculation for each dataset. 


\subsection{DIARETDB1 DATASET RESULT}

The Coye Filter approach is applied on to Diaretdb1. The result for the Diaretdb1 dataset is as following.

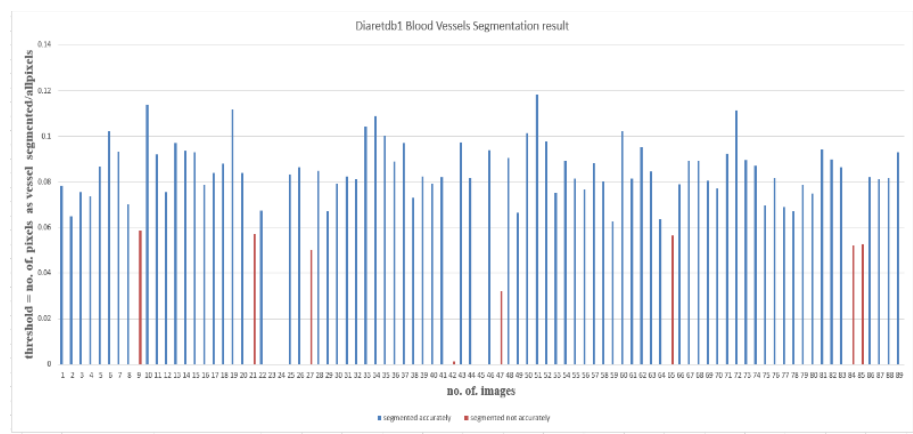

Fig.24. BV Segmentation for Diaretdb1

The Fig.24 shows the accurately segmented image with the blue bars and the images which are not accurately segmented is located by the red bars. The threshold is calculated from 20, 20, 45 manually segmented images respectively.

- Threshold calculation for the Blood Vessels Segmented Image. Consider the image $I, V$ as vector contains the pixels which are belong to the blood vessels such as $V=\{\}$ and $I_{1}$ is vector contains all the pixels from the input image $I$ such as $I_{1}=\{\}$. The threshold for the image $I$ is calculated as following,

$$
\text { Threshold }(t)=V / I_{1}
$$

Table.4. Threshold calculation for Diaretdb1

\begin{tabular}{|l|c|}
\hline \multicolumn{1}{|c|}{ Images } & $\begin{array}{c}\text { Min } \\
\text { Threshold }\end{array}$ \\
\hline $\begin{array}{l}\text { 20 Images from DRIVE training manual } \\
\text { Segmented BV }\left(t_{1}\right)\end{array}$ & 0.0603 \\
\hline $\begin{array}{l}\text { 20 Images from DRIVE test manual } \\
\text { Segmented BV }\left(t_{2}\right)\end{array}$ & 0.0715 \\
\hline $\begin{array}{l}\text { 45 Images from all manual Segmented } \\
\mathrm{BV}\left(t_{3}\right)\end{array}$ & 0.0487 \\
\hline
\end{tabular}

- Global threshold: Threshold is calculated as ratio of number of pixels belongs to vessels to the all pixels of that image. After getting the min threshold for each dataset, global threshold is calculated by again computing the mean for the all computed min threshold. For each input image the threshold is calculated from the Eq.(7) and compare with the global threshold. If the threshold is greater than the global threshold then the image is consider as accurately segmented blood vessels else the image is consider as not accurately segmented blood vessels.

$$
\left(t_{1}+t_{2}+t_{3}\right) / 3=0.0601
$$

Table.5. Blood Vessels Segmentation Accuracy calculation for Diaretdb1

\begin{tabular}{|c|c|}
\hline Parameter & No. of images \\
\hline Segmented Accurately & 78 \\
\hline Segmented not accurately & 11 \\
\hline
\end{tabular}

- Accuracy for Blood Vessels Segmentation $=87.64 \%$. In this way, the accuracy for the other 4 datasets is given in Table. 6 .

Table.6. Accuracy calculation for datasets

\begin{tabular}{|c|c|c|c|c|c|}
\hline Dataset & Total & $\begin{array}{c}\text { Global } \\
\text { Threshold }\end{array}$ & $\begin{array}{c}\text { Segmented } \\
\text { Accurately }\end{array}$ & $\begin{array}{c}\text { Not } \\
\text { Accurately } \\
\text { Segmented }\end{array}$ & Accuracy \\
\hline DRIVE & 20 & 0.0760 & 17 & 3 & $85 \%$ \\
\hline All & 45 & 0.0760 & 35 & 10 & $77.77 \%$ \\
\hline Normal & 30 & 0.0601 & 23 & 7 & $76.66 \%$ \\
\hline Abnormal & 30 & 0.0601 & 23 & 7 & $76.66 \%$ \\
\hline
\end{tabular}

The Global threshold calculation is depend on the dataset. For the Diaretedb1 Normal and Abnormal datasets the global threshold is calculated as mean of the minimum $t$ determined from each manually segmented blood vessels datasets. For the DRIVE and All datasets the global threshold is calculated as the mean of the thresholds of the 45 manually segmented blood vessels images dataset.

\subsection{RGB INTENSITIES ANALYSIS FOR THE RETINAL IMAGES}

The RGB intensities for the ten Retinal healthy Images ROI, are as following.

Table.7. RGB intensities for 10 Retinal healthy Images

\begin{tabular}{|c|c|c|c|}
\hline Image No. & Red intensity & Green intensity & Blue intensity \\
\hline 1 & 255 & 255 & 166 \\
\hline 2 & 255 & 255 & 206 \\
\hline 3 & 255 & 255 & 182 \\
\hline 4 & 255 & 255 & 255 \\
\hline 5 & 255 & 255 & 255 \\
\hline 6 & 255 & 255 & 255 \\
\hline 7 & 255 & 255 & 175 \\
\hline 8 & 255 & 255 & 240 \\
\hline 9 & 255 & 255 & 178 \\
\hline 10 & 255 & 255 & 212 \\
\hline
\end{tabular}

From the Table.7, the average of red, Green and Blue intensities from all the healthy Retinal images ROI is $100 \%$ [17], $100 \%$ and $83.29 \%$ respectively. The RGB intensities for the ten Retinal Glaucoma affected Images ROI, are as following.

Table.8. RGB intensities for 10 Retinal Glaucoma affected Images

\begin{tabular}{|c|c|c|c|}
\hline Image No. & Red intensity & Green intensity & Blue intensity \\
\hline 1 & 255 & 221 & 144 \\
\hline 2 & 255 & 223 & 131 \\
\hline 3 & 255 & 212 & 136 \\
\hline 4 & 255 & 235 & 148 \\
\hline 5 & 255 & 186 & 106 \\
\hline 6 & 255 & 210 & 108 \\
\hline
\end{tabular}




\begin{tabular}{|c|c|c|c|}
\hline 7 & 255 & 238 & 147 \\
\hline 8 & 255 & 209 & 132 \\
\hline 9 & 255 & 255 & 154 \\
\hline 10 & 255 & 255 & 157 \\
\hline
\end{tabular}

From the Table.8, The average of red, Green and Blue intensities from all the Glaucoma affected Retinal images ROI is $100 \%, 88 \%$ and $53.45 \%$ respectively. By observing the Table.7 and the Table. 8 it can be say that, due to the Glaucoma affection, the optic nerve head is get blown up and that's why the average intensities of the Green and Blue channels in the Glaucoma affected ROI is reduces up to the $12 \%$ and $29.84 \%$ respectively.

\section{CONCLUSIONS}

The used of Contrast Limited Adaptive Histogram Equalization method for enhancing the contrast of the Gray image, explores the major arcade of the Blood Vessels with great details through great brightness also the use of the ISODATA method for finding the global threshold for binary conversion lead to extract the continuous as well as non-continuous Blood Vessels with major arcade. The use of the MRF method for segmenting the bright region of the optic disc gives the accurate contour of the bright region. This binary contour is then convoluted with the red and green channel enhanced image for getting the clear bright region with great details for ROI image which is given as input to the Eye Disease Prediction Approach. From the analysis of the healthy Retinal images set found that, The presence of average Red, Green and Blue Channels intensity in the healthy ROI is $100 \%, 100 \%$ and $83.29 \%$ respectively. Where as in Glaucoma affected Retinal images set found that, The presence of average Red, Green and Blue Channels intensity in the Glaucoma affected ROI is $100 \%, 88 \%$ and $53.45 \%$ respectively. The reduction in the intensities of the Green as well as in the Blue Channels is due to the blown up of the Optic nerve head. Due to the blown up of the Optic nerve head the size of the bright region of the Optic Disc is increases but the intensities of the Channels is reduces. There is no effect on the Red Channels but, the average reduction percentage in the Green and in Blue Channels is respectively $12 \%$ and $29.84 \%$.

\section{REFERENCES}

[1] A. Salazar-Gonzalez, "Segmentation of the Blood Vessels and Optic Disk in Retinal Images", IEEE Journal of Biomedical and Health Informatics, Vol. 18, No. 6, pp. 1874-1886, 2014.

[2] Tyler Coye, "A Novel Retinal Blood Vessel Segmentation Algorithm for Fundus Images", Available at: https://in.mathworks.com/matlabcentral/fileexchange/5083 9-a-novel-retinal-blood-vessel-segmentation-algorithm-forfundus-images.

[3] Alan V. Oppenheim, "Discrete-Time Signal Processing", Pearson Education, 1999.

[4] Contrast Limited Adaptive Histogram Equalization, Available at: https://in.mathworks.com/help/images/ref/adapthisteq.html.
[5] X. Zhu, R.M. Rangayyan and A.L. Ells, "Digital Image Processing for Ophthalmology: Detection of the Optic Nerve Head", Synthesis Lectures on Biomedical Engineering, Vol. 6, No. 1, pp. 100-106, 2011.

[6] Chun-Yuan Yu and Shyr-Shen Yu, “Automatic Localization of the Optic Disc Based on Iterative Brightest Pixels Extraction", Proceedings of IEEE International Symposium on Computer, Consumer and Control, pp. 121-124, 2014.

[7] X. Zhu, R.M. Rangayyan and A.L. Ells, "Detection of the Optic Nerve Head in Fundus Images of the Retina using the Hough Transform for Circles", Journal of Digital Imaging, Vol. 23, No. 3, pp. 332-341, 2010.

[8] Alireza Osareh et al., "Comparison of Colour Spaces for Optic Disc Localization in Retinal Images", Proceedings of $16^{\text {th }}$ International Conference on Pattern Recognition, pp. 231-235, 2002.

[9] Shijian Lu, "Automatic Optic Disc Detection using Retinal Background and Retinal Blood Vessels", Proceedings of $3^{\text {rd }}$ International Conference on Biomedical Engineering and Informatics, pp. 412-415, 2010.

[10] C. Sinthanayothin et.al., "Automated Localization of the Optic Disc, Fovea, and Retinal Blood Vessels from Digital Colour Fundus Images", British Journal of Ophthalmology, Vol. 83, No. 8, pp. 902-910, 1999.

[11] Mira Park, Jesse S. Jin and Suhuai Luo, "Locating the Optic Disc in Retinal Images", Proceedings of International Conference on Computer Graphics, Imaging and Visualization, pp. 87-91, 2006.

[12] Thomas Walter and Jean-Claude Klein, "Segmentation of Color Fundus Images of the Human Retina: Detection of the Optic Disc and the Vascular Tree using Morphological Techniques", Proceedings of International Symposium on Biological and Medical Data Analysis, pp. 282-287, 2001.

[13] Muhammad Zubair, Abubakar Yamin and Shoab A. Khan, "Automated Detection of Optic Disc for the Analysis of Retina using color Fundus Image", Proceedings of IEEE International Conference on Imaging Systems and Techniques, pp. 302-307, 2013.

[14] Frank Ter Haar, "Automatic Localization of the Optic Disc in Digital Colour Images of the Human Retina", Master Thesis, Department of Computer Science, Utrecht University, 2005.

[15] Joes Staal, et al., "Ridge-based Vessel Segmentation in Color Images of the Retina", IEEE Transactions on Medical Imaging, Vol. 23, No. 4, pp. 501-509, 2004.

[16] Jae S. Lim, "Two-Dimensional Signal and Image Processing", Prentice Hall, 1990.

[17] J. Liu, et al., "ARGALI: An Automatic Cup-to-Disc Ratio Measurement System for Glaucoma Detection and Analysis Framework", Proceedings of International Conference on Medical Imaging, pp. 663-667, 2009.

[18] Quan Wang, "HMRF-EM-Image: Implementation of the Hidden Markov Random Field Model and its ExpectationMaximization Algorithm", Proceedings of International Conference on Computer Vision and Pattern Recognition, pp. 1-4, 2012. 